\title{
Micotossine: prevenire è molto, molto meglio che curare
}

\author{
Giuseppe Firrao, Emanuela Torelli, Emanuela Gobbi \\ Dipartimento di Scienze Agrarie ed Ambientali, Università di Udine, Italy
}

\begin{abstract}
Food contamination with fungal secondary metabolites has been a major limit for population wellness and development in the past. Although today social protection against this plague has been established in most countries, there are still many areas in the world where mycotoxin contamination causes illness and even death, due to the lack of healthy conditions for the storage of commodities. Information and knowledge are the main weapons available to contrast the danger of food contamination by mycotoxins. In several instances, information about the severity of the issue alone would have been effective in preventing cases of acute intoxications. Prevention is the winning strategy not only at the level of the end consumer, but also as far as the production is concerned. Thanks to the advances in the understanding of fungal epidemiology and mycotoxin synthesis, strategies for the prevention or at least for the limitation of mycotoxin contaminations have been developed in recent years. As far as cereal production in Italy is concerned, proper storage conditions are now widely adopted, and protocols for good practice in cultivation are being followed by an increasing number of producers. In addition, rapid methods for the early detection of contaminants and quality assessment have been developed and, when introduced in the routine field operation, may lead to substantial improvements of product quality.
\end{abstract}

\section{Riassunto}

La contaminazione di alimenti con metaboliti secondari di origine

Correspondence: Giuseppe Firrao, Dipartimento di Scienze Agrarie ed Ambientali, Università di Udine, via Scienze 208, 33100 Udine.

Tel. +39.0432 .558531 - Fax: +39.0432 .558501 .

E-mail: firrao@uniud.it

Key words: mycotoxins, contamination, prevention.

Parole chiave: micotossine, contaminazione, prevenzione.

Received for publication: 1 March 2011.

Accepted for publication: 30 April 2011.

Società rappresentata nell'ambito AISSA: Società Italiana di Patologia Vegetale - SIPAV

(C) Copyright G. Firrao et al., 2011

Under no circumstances figures can be used without prior written consent of the copyright owner.

Licensee PAGEPress, Italy

Italian Journal of Agronomy 2011; 6(s2):e4

doi:10.4081/ija.2011.6.s2.e4

This work is licensed under a Creative Commons Attribution NonCommercial 3.0 License (CC BY-NC 3.0). fungina ha scandito la storia dell'umanità con episodi famosi e spesso tragici. Meno noto è che ancora oggi in molte zone del mondo si continua a soffrire e persino a morire a causa delle micotossine, principalmente per le inidonee condizioni di conservazione degli alimenti. Le numerose patologie legate al consumo di materiale contaminato sono oggi rese anche più gravi dalla frequente associazione col consumo di alcool o con la diffusione endemica di virus che compromettono la funzionalità epatica, rendendo in alcune zone del mondo la problematica dell'alimentazione un'emergenza più per gli aspetti qualitativi che per quelli quantitativi. Le armi più efficaci per contrastare la diffusione delle micotossine sono il sapere e la conoscenza. Nei casi più importanti di intossicazione cronica ed acuta da micotossine registrati a livello mondiale il solo informare la popolazione dell'esistenza del problema sarebbe stato sufficiente per l'adozione da parte della stessa di elementari misure preventive che permettono di ridurre drasticamente l'impatto sulla salute. Il principio della prevenzione si estende oltre il momento del consumo dell'alimento ed investe anche le fasi produttive e commerciali. A livello produttivo la conoscenza della problematica, con quella necessaria multidisciplinarietà che caratterizza le nostre scienze agrarie, permette di definire le modalità operative attraverso le quali prevenire 0 almeno di limitare l'infezione delle colture da parte di funghi tossigeni, riducendo sostanzialmente la produzione di micotossine in campo. Per i cereali sono ormai patrimonio acquisito le modalità con le quali condurre opportunamente, al momento della raccolta, le operazioni di essiccamento e di immagazzinamento per evitare lo sviluppo di muffe e dei loro metaboliti. Altrettanto rilevanti sono gli accorgimenti tecnici necessari per la precoce individuazione delle partite ad alto contenuto di micotossine che permettano di escludere dalle linee produttive i lotti più contaminati. In tutti questi settori la ricerca, in particolare italiana, è stata estesa e produttiva, non solo nel rendere diffusamente disponibili gli strumenti tecnici, ma soprattutto nell'identificazione dei fattori di rischio, prima ed indispensabile nozione per l'approntamento delle strategie preventive. L'inosservanza di metodi preventivi si traduce nell'immissione nella catena alimentare di contaminanti che compromettono la sicurezza sanitaria degli alimenti e ne riducono sostanzialmente, ed irreparabilmente, il valore commerciale.

\section{Introduction}

Numerosi prodotti vegetali utilizzati per l'alimentazione umana 0 animale possono fungere da substrato per lo sviluppo di microorganismi fungini. In molte occasioni alla crescita fungina si accompagna la produzione di metaboliti secondari, detti micotossine, dalle preoccupanti caratteristiche di tossicità acuta e cronica, attività carcinogena e immunodepressiva. La contaminazione da micotossine é stata messa in diretta relazione con fenomeni di intossicazione del bestiame e di insorgenza di gravi patologie nell'uomo (Nelson et al., 1993). Per questo motivo da molti anni ormai a diversi livelli le competenti autorità stabiliscono precisi limiti alla commercializzazione e al consumo, sia umano che animale, di materiale vegetale che risulti contaminato da alcune micotossine. 
Tuttavia, difficilmente si ha una chiara percezione della rilevanza della protezione di cui attualmente godiamo. Per noi ormai è radicato il concetto ampio di qualità del cibo, comprensivo sia degli aspetti nutrizionali ed organolettici che anche della sua salubrità. Si tende spesso ad associare la qualità del cibo con il benessere e secondo una certa linea di pensiero il problema della fame nel mondo dovrebbe essere affrontato fornendo cibo sufficiente e nutrizionalmente equilibrato a tutti gli essere umani, preoccupandosi solo in un secondo momento della sicurezza di questo cibo. E' una concezione in realtà dominante, come testimonia il fatto facilmente riscontrabile che il livello di controllo sociale sulla sicurezza del cibo risulti in effetti una funzione diretta del livello di benessere di una società. La storia tuttavia ci dice che le cose non stanno esattamente così, che collegare qualità degli alimenti ad una generica idea di superfluo benessere è assai riduttivo e che, viceversa, la qualità del cibo ha un impatto sostanziale sulla sopravvivenza e sulla socialità.

Ai tempi in cui le popolazioni malnutrite si trovavano nel Nord del mondo, per esempio, la contaminazione di alimenti con metaboliti secondari di origine fungina ne ha tragicamente scandito la storia con episodi di cui solo più recentemente si sono comprese e pienamente apprezzate le cause. Durante la seconda guerra mondiale, in una situazione in cui il livello della vita delle popolazioni rurali in Russia garantiva con difficoltà la sopravvivenza, per diversi anni, a causa di un andamento climatico sfavorevoli ed alle oggettive difficoltà imposte dalla guerra, i cerali, tipicamente la segale, non furono tempestivamente raccolti e rimasero a lungo, in alcuni casi l'intero inverno, sotto la neve. In queste condizioni è frequente lo sviluppo di un fungo, Fusarium sporotrichioides, che produce la tossina T-2. A causa della fame, i contadini russi raccolsero ed utilizzarono cerali contaminati rimanendo vittime di quella che oggi è chiamata aleukia tossica alimentare, una sindrome spaventosa che colpisce l'apparato digerente provocando nausea, vertigini, fino a diffuse ulcerazioni ed infine la morte nei casi gravi. Le dimensioni di questo fenomeno furono enormi, con un livello di mortalità che toccò in alcune aree punte del $60 \%$. Nelle zone colpite circa un terzo dei membri delle famiglie che si erano nutrite di cereali contaminati non sopravvisse. Si stima che le morti siano state alcune centinaia di migliaia, per una situazione protrattasi per anni a causa della sostanziale incapacità della popolazione di associare la sindrome all'assunzione di cereali ammuffiti, che avvenne solo nel 1968 (Marasas e Nelson, 1987b).

È significativo quanto la situazione descritta sia simile a quella attuale nei paesi dove la fame si è spostata, dal Nord al Sud. Gli anni 2004-2005 hanno visto svilupparsi in Kenya una delle più estese aflatossicosi di cui si abbia memoria, con 317 casi di intossicazione acuta riportati e 125 decessi (Shephard, 2008). In questo caso il cereale imputato è il mais. A causa del clima inusualmente piovoso il raccolto fu scarso ed estremamente umido, ed in aggiunta la granella non fu immagazzinata nei granai per impedire i furti, frequenti causa della scarsità di cibo, ma direttamente nelle abitazioni che nella stagione piovosa sono mal ventilate ed umide a causa dei tetti di fogliame che non impediscono totalmente alla pioggia di penetrare. In questo caso, in condizioni climaticamente opposte a quelle della Russia negli anni 40, con una stagione calda e umida soprattutto nel 2004 ma anche nel 2005, il fungo che si sviluppò fu Aspergillus flavus e la tossina prodotta l'aflatossina. In Kenia il mais era commerciato ad un prezzo compreso tra i 16 and 22 Scellini Kenyani per chilo, ma esisteva un mercato anche per il mais di seconda qualità, sostanzialmente ammuffito, che veniva venduto ad un prezzo di circa la metà, normalmente per l'alimentazione animale. Nel 2004 a causa della scarsità del raccolto il prezzo del mais subì un forte aumento ed il mais ammuffito sostituì in misura molto estesa il mais sano nelle mense rurali. L'indagine che seguì rivelò che più del $55 \%$ dei campioni di mais presenti sul mercato avevano un tenore di aflatossine maggiore del livello considerato la soglia di riferimento nella normativa $(20 \mathrm{ng} / \mathrm{g})$ ed il $7 \%$ ne conteneva quantità eccezionalmente elevate (>1000 ng/g; Lewis et al., 2005).

Per quanto evidentemente questi fenomeni siano di proporzioni notevoli, il resoconto sui fenomeni di tossicità acuta non è che l'estrema punta dell'iceberg della problematica che le micotossicosi rappresentano per la sopravvivenza delle popolazioni povere. L'aflatossina non soltanto è estremamente tossica con effetti gravi a livello epatico, ma è anche il più potente carcinogeno naturale di cui si abbia notizia. Le citate popolazioni del Kenya non associano i fenomeni di intossicazione al consumo di cereali ammuffiti, e finché una diffusa opera di sensibilizzazione non sarà portata a termine e la presenza di un mercato dei cereali ammuffiti verrà tollerata, queste popolazioni continueranno ad avvelenarsi con una sostanza che a livello cronico debilita il sistema immunitario e le funzioni epatiche. Il sinergismo della intossicazione cronica di aflatossina con la debilitazione fisica dovuta alla scarsa alimentazione, con la diffusione endemica di malattie che interessano il sistema immunitario e la funzione epatica ed infine con l'abuso di sostanze alcooliche, ha effetti gravissimi sulla capacità di sopravvivenza delle popolazioni interessate.

L'effetto di una intossicazione cronica su una popolazione è sicuramente molto più difficile da valutare di quanto non sia raccogliere dati su un caso di diffusa intossicazione acuta, ma è in realtà molto più rilevante per la popolazione nel suo complesso. Valutazioni storiche sono state effettuate per quanto riguarda l'impatto dei metaboliti di un altro fungo, Claviceps purpurea, causa di una sindrome chiamata ergotismo. Il fungo è famoso per la produzione di sclerozi (noti come segale cornuta), che contengono diversi alcaloidi, alcuni con interessanti proprietà medicali altri con struttura affine ad alcuni neurotrasmettitori come l'acido lisergico e per questo dotati di proprietà allucinogene. L'ergotismo è infatti principalmente famoso per la rilevanza sociale che attiene all'effetto degli alcaloidi sulla psiche e sulla socialità, non dico per arrivare a quanto alcuni affermano che abbia avuto un ruolo nella rivoluzione francese, ma certamente per esempio per quanta riguarda il famoso processo alle streghe di Salem (Massachusetts, USA), nel 1692, che si concluse con 27 condanne a morte, di cui 19 effettivamente eseguite, di giovani donne che f,urono ritenute indemoniate a causa degli effetti di convulsioni e di allucinazioni, e che è stato dimostrato essere soggetti particolarmente esposte per la loro età e per la posizione dei loro campi di cerali, ad intossicazione di ergot in anni in cui le condizioni climatiche furono particolarmente favorevoli allo sviluppo del fungo. Ma ancora si tratta di casi di intossicazioni acute, come è stata, tanto per fare ancora un parallelo con l'Africa di oggi, l'epidemia di ergot nel Walla, in Etiopia. Dopo due anni successivi di siccità le culture di orzo locale, tipico alimento della popolazione, sono state invase da avena selvatica fortemente infettata da Claviceps purpurea. Nella primavera del 1978 su una popolazione di 41.900 abitanti sono stati registrati 93 casi di ergotismo cancrenoso, con un totale di 47 morti (Marasas e Nelson, 1987a).

Al di là della rilevanza di questi casi di intossicazione acuta, il punto che interessa qui sottolineare è che i metaboliti contenuti negli sclerozi di $C$. purpurea sono fortemente debilitanti per la salute in modo cronico, soprattutto per l'effetto vasocostrittorio che compromette la circolazione sanguigna. Oggi vi sono motivi per ritenere che la grande esplosione demografica avvenuta nell'Europa centro settentrionale negli anni tra il 1750 ed il 1850 sia fondamentalmente dovuta alla introduzione massiccia della patata con la conseguente riduzione della segale nell'alimentazione. La consuetudine all'ingestione in concentrazioni subtossiche di metaboliti di $C$. purpurea contenuti nel pane si traduceva in uno stato di anemia, infertilità, immunodepressione e generalizzata debilitazione fisica fortemente limitanti le capacità di sopravvivenza della popolazione (Matossian, 1989).

È interessante notare come molte zone dell'Europa dell'Est, dove la patata entrò nell'alimentazione con molto ritardo, non furono oggetto di questa esplosione demografica. Fino alla fine dell'ottocento, la segale cornuta contaminava in Ucraina, Bielorussia e in varie provincie 
della russia (Ivanovo, Yaroslavl, Moscow, Smolensk, Voronezh) negli anni con andamento climatico predisponente l'infezione, il 75\% delle spighe, mentre la mortalità infantile non scendeva sotto il $26 \%$, quando in Francia era del 16\%, in Italia del 15\% ed in Irlanda, dopo la scoperta che il rame fermava la peronospora, era addirittura del $10 \%$.

L'esercito russo, fin dal 1882, aveva incaricato commissioni di studiosi che avevano chiarito che non c'è modo di eliminare gli alcaloidi dell'ergot dalla farina e quindi procedeva a seri controlli preventivi prima dell'acquisto. Quello che rimaneva alle popolazioni rurali era quindi in genere un prodotto decisamente contaminato in tutti gli anni in cui il clima era favorevole a $C$. purpurea. L'introduzione della patata cosituì una benedizione per queste popolazioni. Ma il concetto fondamentale fu sviluppato e ribadito dall'azione dell'esercito russo cui si accennava: le conseguenze dell'ingestione di micotossine sono talmente gravi, e contemporaneamente così subdolamente difficili da individuare, che vale la spesa sostenere uno sforzo significativo per impedirla, e la contaminazione è così difficile da eliminare che è decisamente conveniente fare ogni possibile sforzo per prevenirla (Matossian, 1989). Queste tesi si sono dimostrati estremamente valide anche alla luce delle più recenti indagini epidemiologiche. Prevenzione, nel contesto di agricoltura industriale che oggi pratichiamo, si traduce fondamentalmente in tre concetti, che esporremo in riferimento a quello che oggi è il problema forse non più grave dal punto di vista tossicologico ma certamente il più diffuso ed il più complesso nelle regioni del Nord Italia, e cioè la contaminazione del mais.

I cereali da granella, la frutta secca, il caffé sono alimenti particolarmente soggetti alla contaminazione da micotossine. Nell'Italia del Nord forte preoccupazione desta in questi ultimi anni l'intensità della contaminazione del mais da fumonisina, una potente micotossina prodotta da Fusarium verticillioides ed alcune altre specie minori, mentre resta alta l'attenzione verso la contaminazione da aflatossina, pericolosamente trasmessa attraverso la catena alimentare e prodotta in larga misura da Aspergillus flavus che occasionalmente, quando l'andamento stagionale è favorevole, contamina gravemente il mais. Questo cerale, centrale nel sistema agricolo norditaliano, è dunque oggetto di diverse contaminazioni di natura micotica. Al momento della raccolta, dell'essiccamento e dell'immagazzinamento congiunto di produzioni di diversa origine, si verifica la contaminazione di grandi volumi di prodotto da parte di una quantità, magari anche limitata, di granella fortemente infetta. Le micotossine che finiscono disperse in grandi volumi di prodotto non possono più esserne convenientemente rimosse, essendosi dimostrate estremamente resistenti a trattamenti chimici e termici (Nelson et al., 1993). Le azioni di prevenzione della contaminazione sono quindi tanto più efficaci ed economiche quanto più intervengono nelle fasi iniziali della filiera produttiva e commerciale.

La prima e fondamentale azione di prevenzione, che è quella del corretto essiccamento ed immagazzinamento, è ormai un fatto acquisito per la nostra agricoltura. Possiamo dire di essere stati definitivamente vaccinati nel 2003 in occasione della breve ma intensa crisi aflatossina nel periodo autunnale. Nel 2003 la stagione estiva fu particolarmente calda, cosa che produsse un'accelerazione della maturazione del mais che portò frequentemente in raccolta la granella a valori di umidità inusualmente bassi, vicini a quelle di conservazione (14\%) (Blandino e Reyneri, 2004). Con quei valori alcuni operatori decisero di immagazzinare la granella senza passare dall'essiccatoio, ma l'umidità residua, l'elevata temperatura e le trascorse condizioni favorevoli allo sviluppo di un cospicuo inoculo di Aspergilli fecero il resto. Quando il prodotto passò nella razione delle bovine vennero immediatamente riscontrati nel latte valori di aflatossina M1 sopra la norma: le cause vennero identificate ed isolate prontamente, ma certo vi fu un danno economico ed un impatto sociale significativi, che restano di monito per l'esecuzione delle pratiche corrette.

La seconda importante azione preventiva consiste nell'impedire la crescita dei funghi micotossigeni e la produzione di tossine durante la coltivazione. La ricerca e la sperimentazione agronomica in questi ultimi anni hanno dato indicazioni chiare su come è opportuno intervenire tecnicamente sulla pratica colturale per produrre granella di mais di migliore qualità sanitaria. Numerosi interventi agronomici si sono rivelati significativamente correlati con la riduzione del tenore in micotossine. Conseguentemente, sono stati messi a punto protocolli di buona pratica colturale che definiscono le principali e più efficaci linee di condotta per la produzione di mais sano (Snidaro e Paliotti, 2002), e sono stati fatti progressi nello sviluppo di processi di simulazione, che permettono sulla scorta dell'interfacciamento tra risultati analitici e dati meteorologici e geografici di produrre delle mappe di rischio con alto valore predittivo, utili a concentrare gli interventi nelle zone e nei periodi che lo richiedono (Battilani et al., 2008). Tuttavia la produzione di qualità rimane dipendente da una profonda ristrutturazione dell'intera filiera e da una forte azione di sensibilizzazione e formazione di tutti gli operatori, garantita da una rete scientifica, tecnica e divulgativa che oggi si sta sviluppando. Qualità significa spesso rinuncia a rese/ettaro e quindi a redditività ormai consolidate per gli imprenditori agricoli, e applicazione di ulteriori vincoli alla loro attività non ancora riconosciuti economicamente dal mercato nei meccanismi che regolano il valore aggiunto delle produzioni agroalimentari. Dove il clima permette una prolungata permanenza in campo del mais in attesa del momento migliore per il conferimento del prodotto in funzione del maggior prezzo di mercato, unitamente a oggettive carenze infrastrutturali di alcuni centri di raccolta ed essiccazione, si assiste ancora a raccolte molto tardive accompagnate da prolungate fasi di accumulo del prodotto prima dell'inizio del processo di essiccazione, troppo spesso contraddistinte da elevate contaminazioni con micotossine.

È infine la terza azione che costituisce il metodo più efficace per contrastare l'impatto dannoso delle micotossine sulle società: impedire l'ingresso delle tossine nella catena alimentare. Il problema delle micotossine che contaminano cronicamente la nostra produzione, quali le fumonisine, potrà essere in gran misura risolto, nel lungo periodo, con l'adeguamento della produzione agli accorgimenti definiti dalla ricerca agronomica, purché questa sia attivamente trasferita alla pratica colturale. Al momento, grazie alla consapevolezza e l'impegno di molti tra i produttori e gli operatori, i risultati cominciano a vedersi, anche se rimane vero che dove la riduzione del contenuto di tossine entro i limiti consentiti richiede interventi che limitano sensibilmente le quantità prodotte, la mancanza della capacità tecnica di valutare qualitativamente la granella al momento del conferimento impedisce il controllo utile a incentivare la buona pratica. E' ovvio che se la qualità richiede costo ed impegno, ma non può essere premiata, è difficile ottenere risultati. Si deve inoltre tener presente che quando il rispetto delle buone pratiche, soprattutto nelle fasi di produzione, è impedito o reso difficoltoso nella sua applicazione da fattori climatici e casuali, è doveroso immaginare che anche negli scenari futuri più favorevoli, in cui gli operatori siano attivamente impegnati nello sforzo per ridurre la contaminazione, permangano situazioni locali contraddistinte da contaminazioni non insignificanti. Ma convivere con la contaminazione di alcune micotossine a diffusione endemica, come per esempio le fumonisine, sarebbe possibile con l'indirizzamento delle partite ad utilizzi diversi in base al loro grado di contaminazione. E' stato messo in luce come la tollerabilità dei diversi animali alla fumonisina sia diversa, e che la fumonisina non si trasmette lungo la linea alimentare. Per questo la definizione di linee di lavorazione separate permetterebbe di indirizzare mais a diverso grado di contaminazione verso diversi utilizzi. Il mais meno contaminato (meno di $2 \mathrm{ppm}$ ) dovrebbe essere indirizzato al consumo umano diretto, quello mediamente contaminato può essere utilizzato per l'alimentazione di bovini e suini, mentre quello maggiormente contaminato può essere utilizzato per l'alimentazione dei polli, il cui metabolismo tollera fino a $100 \mathrm{ppm}$ senza ripercussioni fisiologiche né accumulo nella carne. Il mais che risulta avere un contenuto in micotossine fuori dalla norma dovrà essere necessariamente 
eliminato dalla filiera agroalimentare. Una utile destinazione alternativa è l'utilizzo a fini energetici. Sono già in commercio caldaie specifiche per cereali (mais ma anche frumento) capaci di utilizzare il mais sia in granella che eventualmente pelletato (raccolto con tutta la pianta) con elevati rendimenti. Con un potere calorifico stimabile in circa $4000 \mathrm{kcal} / \mathrm{kg}$ al 14\% di umidità il mais risulta oggi una fonte di energia termica più conveniente del gasolio.

La definizione degli aspetti della tecnologia produttiva rilevanti per l'ottenimento di un materiale sano deve dunque essere coadiuvata dal potenziamento delle metodologie di campionamento ed analisi, indispensabili sia per il controllo e la verifica della buona pratica che per l'indirizzamento delle partite a processi produttivi distinti. In questi anni sulla la diagnosi precoce e la valutazione del rischio associato alle partite la ricerca è stata intensa, con lo sviluppo di nuovi metodi basati su tecnologie d'avanguardia quali la spettroscopia infrarossa (Berardo et al., 2005), il naso elettronico (Gobbi et al., 2011), l'analisi dell'immagine (Firrao et al., 2010) ed il campionamento aereobiologico (Torelli et al., 2010).

Grazie all'attuazione delle tre azioni preventive, che consentano di pervenire alla produzione di prodotti con contenuto di micotossine sempre minore, di individuare tempestivamente le partite maggiormente contaminate per poterle dirigere verso linee di lavorazione differenziate e di prevenir la formazione di micotossine durante l'immagazzinamento siamo oggi in Italia, come negli altri paesi industrializzati, fieri di essere in grado di provvedere ai fabbisogni alimentari del Paese con prodotti privi di quei contaminanti che nei secoli passati hanno limitato lo sviluppo ed il benessere della popolazione.

\section{Bibliografia}

Battilani P., Pietri A., Barbano C., Scandolara A., Bertuzzi T., Marocco A., 2008. Logistic regression modeling of cropping systems to predict fumonisin contamination in maize. J. Agric. Food Chem. 56:10433-10438.

Berardo N., Pisacane V., Battilani P., Scandolara A., Pietri A., Marocco A., 2005. Rapid detection of kernel rots and mycotoxins in maize by near-infrared reflectance spectroscopy. J. Agric. Food Chem. 53:
8128-8134.

Blandino M., Reyneri A., 2004. 2003: una campagna a rischio per le aflatossine. Inform. Agr. 6 49-49.

Firrao G., Torelli E., Gobbi E., Raranciuc S., Bianchi G., Locci R., 2010. Prediction of milled maize fumonisin contamination by multispectral image analysis. J. Cereal Sci. 52:327-330.

Gobbi E., Falasconi M., Torelli E., Sberveglieri G., 2011. Electronic nose predicts high and low fumonisins contamination in maize cultures. Food Res. Int. 44:992-999.

Lewis L., Onsongo M., Njapau H., Schurz-Rogers H., Luber G., Kieszak S., Nyamongo J., Backer L., Dahiye A.M., Misore A., 2005. Aflatoxin contamination of commercial maize products during an outbreak of acute aflatoxicosis in eastern and central Kenya. Environ. Health Persp. 113:1763-1767.

Marasas W.F.O., Nelson P.E., 1987a. Ergotism. In: W.F.O. Marasas and P.E. Nelson (eds.) Mycotoxicology. Introduction to the Mycology, Plant Pathology, Chemistry, Toxicology, and Pathology of Naturally Occurring Mycotoxicoses in Animals and Man. Pennsylvania State University Press, PA, USA, pp 19-22.

Marasas W.F.O., Nelson P.E., 1987b. Hemmorhagic syndrome. In: W.F.0. Marasas and P.E. Nelson (eds.) Mycotoxicology. Introduction to the Mycology, Plant Pathology, Chemistry, Toxicology, and Pathology of Naturally Occurring Mycotoxicoses in Animals and Man. Pennsylvania State University Press, PA, USA, pp 41-44.

Matossian M.K., 1989. Poisons of the past: molds, epidemics and history. Yale University Press, New Haven, C0, USA.

Nelson P.E., Desjardins A.E., Plattner R.D., 1993. Fumonisins, mycotoxins produced by Fusarium species - biology, chemistry, and significance. Annu. Rev. Phytopathol. 31:233-252.

Shephard G.S., 2008. Impact of mycotoxins on human health in developing countries. Food Addit. Contam. A 25:146-151.

Snidaro M., Paliotti P., 2002. Rinnovare la tecnica di coltivazione del mais per rinnovare la qualità della granella. Notiziario ERSA 1:1517.

Torelli E., Gubiani R., Firrao G., Cividino S., Locci R., Gobbi E., 2010. Air analysis in the assessment of fumonisin contamination risk in maize. J. Sci. Food Agric. 90:641-649. 